\title{
Polymer optical waveguides with reduced in-plane bend loss for electro-optical PCBs
}

\author{
Richard Pitwon $^{* a}$, Callum Smith ${ }^{\mathrm{b}}$, Kai Wang ${ }^{\mathrm{c}}$, Jasper Graham-Jones ${ }^{\mathrm{d}}$, David R. Selviah ${ }^{\mathrm{c}}$, Markus \\ Halter $^{\mathrm{e}}$, Alex Worrall ${ }^{\mathrm{a}}$ \\ ${ }^{a}$ Xyratex Technology Ltd, 1000-40 Langstone Technology Park, Havant, Hampshire, PO9 1SA, \\ $\mathrm{UK}$; \\ ${ }^{\mathrm{b}}$ School of Physics and Astronomy, University of Southampton, Highfield, Southampton, SO17 \\ 1BJ, UK; \\ ${ }^{c}$ Department of Electronic and Electrical Engineering, University College London (UCL), London, \\ WC1E 7JE, UK; \\ ${ }^{\mathrm{d}}$ School of Marine Science \& Engineering, Plymouth University, Drake Circus, Plymouth, PL4 \\ 8AA,UK; \\ ${ }^{\mathrm{e}}$ Markus Halter, Vario-optics AG, Mittelbissaustrasse 7, CH-9410 Heiden / Switzerland
}

\begin{abstract}
In-plane bend loss represents the greatest commercial inhibitor to deploying multimode optical waveguides on densely populated electro-optical printed circuit boards $(\mathrm{OPCB})$ as the minimum bend radii currently possible are too large to be practical in common designs. We present a concept and fabrication method for creating novel polymer optical waveguide structures with reduced bend losses to enable higher density routing on an OPCB. These nested core waveguide structures comprise a core surrounded by a thin shell of cladding, which allows for two-fold modal containment by first a conventional low refractive index contrast (LIC) boundary followed by a secondary high refractive index contrast (HIC) boundary. The purpose of this is to reduce the in-plane bend losses incurred on tightly routed optical channels, while not incurring prohibitive dispersion, sidewall scattering and optical crosstalk penalties.

We have designed and fabricated nested core multimode polymer waveguides, evaluated their performance in comparison to conventional step-index waveguides and simulated these structures using the beam propagation method. Preliminary results are presented of our measurements and simulations.
\end{abstract}

Keywords: Optical waveguides, optical waveguide bends, optical bend loss, nested waveguides, polymer waveguides

\section{INTRODUCTION}

One of the key challenges in designing polymer waveguide based electro-optical printed circuit boards (OPCBs) is providing an optical waveguide interconnect [1-3] pattern that can meet the stringent routing requirements inherent to navigating densely populated printed circuit board layouts while complying with the practical optical design constraints for multimode waveguides. In-plane bends are an inextricable requirement in complex waveguide layouts on OPCBs, however, practical values for optimum multimode waveguide bend radii range from $15 \mathrm{~mm}$ to $20 \mathrm{~mm}$, whereby the bend loss is minimized with respect to arc length and bend radius [4-6]. From a design point of view, it would be very difficult to accommodate bend radii that large within a high density PCB layout, especially where high numbers of optical waveguides are involved.

*rpitwon@xyratex.com; phone +44 (0)23 9249 6715; xyratex.com 
In particular when considering the need for commercially viable design and fabrication techniques for embedded stepindex optical waveguides in printed circuit boards, one must operate within a limiting trade-off space, which includes insertion loss, signal dispersion, refractive index difference between core and cladding, waveguide size, misalignment tolerance, minimum bend radius, maximum waveguide length and sidewall roughness [7-10].

In this paper we present preliminary results of research and development on novel multimode polymer waveguide structures with reduced transitional in-plane bend losses to allow denser and more tightly routed optical channel layouts on an OPCB. While conventional polymer waveguides are comprised of a higher refractive index core, which is fully surrounded by a lower index cladding [11-13], the fabrication of the nested core waveguides involves a second patterning step whereby the cladding is structured around the core, such as to create a secondary higher index contrast boundary near the primary low index contrast core-cladding boundary.

We designed and fabricated a waveguide testbed to allow evaluation of how nested core waveguides can enhance optical modal confinement along different waveguide geometries. We also present BPM simulation results of a tightly routed cascaded waveguide structure in which bend transition losses on the proposed nested core waveguide structures are shown to be reduced compared to conventional waveguides.

This is the first time the low index contrast (LIC) - high index contrast (HIC) waveguide structure has been deployed on the larger scale associated with multimode polymer waveguides.

\section{FABRICATION OF NESTED CORE MULTIMODE POLYMER WAVEGUIDES}

\subsection{Nested core waveguide geometry}

The proposed "nested core" waveguide structures comprise a transparent core surrounded by a thin shell of cladding, the thickness of which ranges from $10 \%$ to $40 \%$ of the core width, which has been chosen primarily for ease of fabrication. As the purpose of these structures is to enhance confinement of propagating optical modes in the plane of the waveguide axis it is important that the size, alignment and surface features of the shell is tightly controlled on the sides of the core (Figure 1).

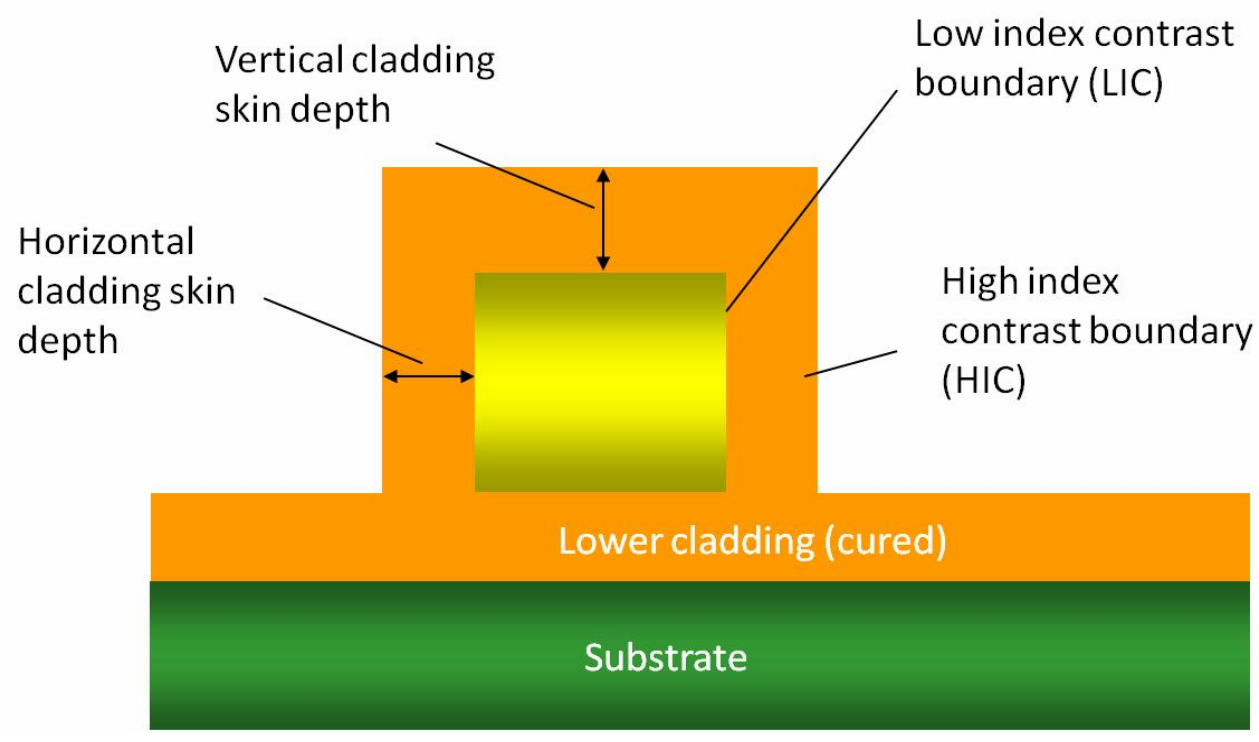

Figure 1: Cross-section of nested core waveguide structure with outer cladding arranged symmetrically around core

We define the parameter Horizontal Cladding Skin Depth (HCSK), which is the horizontal distance between the first LIC core-cladding boundary and the second HIC cladding-air boundary and the parameter Vertical Cladding Skin Depth (VCSK), which is the vertical distance between the first LIC core-cladding boundary and the second HIC cladding-air boundary. The relative importance of either parameter depends on the plane in which the waveguide bends occurs. For in-plane bends (e.g. where bends occur in the plane of the PCB) the HCSK is more relevant, while for outof-plane bends (e.g. where the waveguides are part of a flexible substrate, which itself is bent around on itself) the VCSK is more important. Low Index Contrast (LIC) is defined as the refractive index difference between core and 
cladding. High Index Contrast (HIC) is defined as the refractive index difference between the $1^{\text {st }}$ cladding and the $2^{\text {nd }}$ cladding. In the case of a simple nested core waveguide as shown the $2^{\text {nd }}$ cladding is air.

\subsection{Photolithographic fabrication process}

The nested core waveguide structures were fabricated lithographically by Vario-optics AG. The photolithographic fabrication process is outlined in Figure 2.
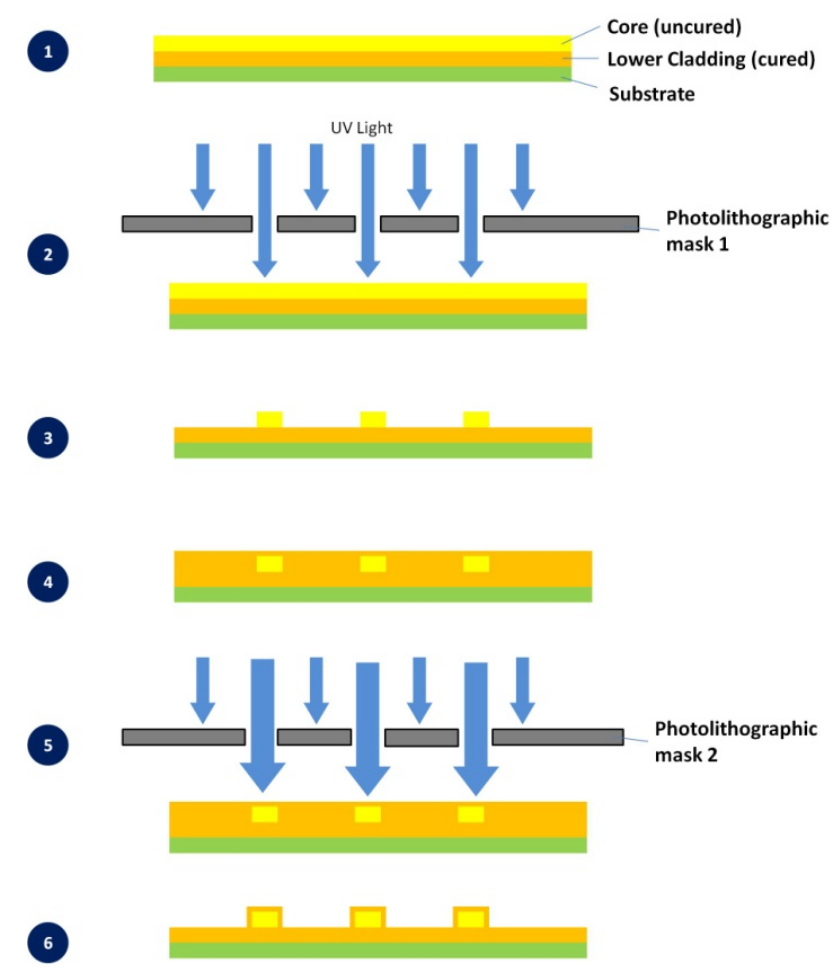

Figure 2: Photolithographic fabrication process for first order nested core waveguides

Different layers of photosensitive polymer are deposited by doctor blading, which allows the uniformity of the liquid polymer surface to be significantly improved compared to the surface roughness of the substrate on which it is deposited. Polymer layer thickness variation of $\pm 5 \mu \mathrm{m}$ of layer thickness can be achieved this way.

Starting with the deposition of liquid photosensitive polymer layer onto a pre-cured lower cladding layer (Step 1), multimode $50 \mu \mathrm{m}$ waveguides were patterned by selective exposure of the core layer to actinic radiation through a high resolution lithographic mask (Step 2). The uncured sections of the core layer were then removed through cleaning or etching with appropriate developing chemicals revealing the cured waveguide cores (Step 3). Another layer of lower refractive index cladding material was then deposited over and around the cores and doctor-bladed with the distance between the top core surface and top cladding surface at least larger than $10 \%$ of the core width i.e. $>=$ cladding skin depth (Step 4).

A second photolithographic mask with the same pattern as the first mask, but with wider openings, was precisely aligned over the existing core pattern and a second exposure carried out (Step 5). These wider openings allow wider secondary waveguides to be cured in the cladding material over the first waveguide cores, giving rise to lateral cladding shells surrounding the core, hence the term nested core waveguides. It is crucial that at least the secondary mask is aligned with high accuracy to the waveguide features in order to ensure that the cladding shells are symmetrically arranged around the original waveguide core. The masks were aligned using micro-stages allowing an alignment accuracy of less than $5 \mu \mathrm{m}$ to be achieved. 
Following a second development process to remove the uncured cladding material the nested core waveguides are revealed (Step 6).

One of the most crucial requirements for the lithographic fabrication process is controlling the surface roughness of the side walls, which should, on a conventional waveguide be below a tenth of the propagating wavelength $(<85 \mathrm{~nm})$. Therefore, the process of developing away uncured polymer is critical, because it significantly influences the scattering losses in the waveguides. Side wall surface quality is even more critical for the outer high index contrast (HIC) boundary as the scattering losses will be amplified in comparison to the conventional low index contrast (LIC) boundary.

Figure 3 shows the cross-section of a first order nested core waveguide illuminated with white light. The small "skirts" of core material visible around the bottom of the inner core are a typical feature in polymer waveguide fabrication and are a contributor to cross-talk in conventional waveguides.

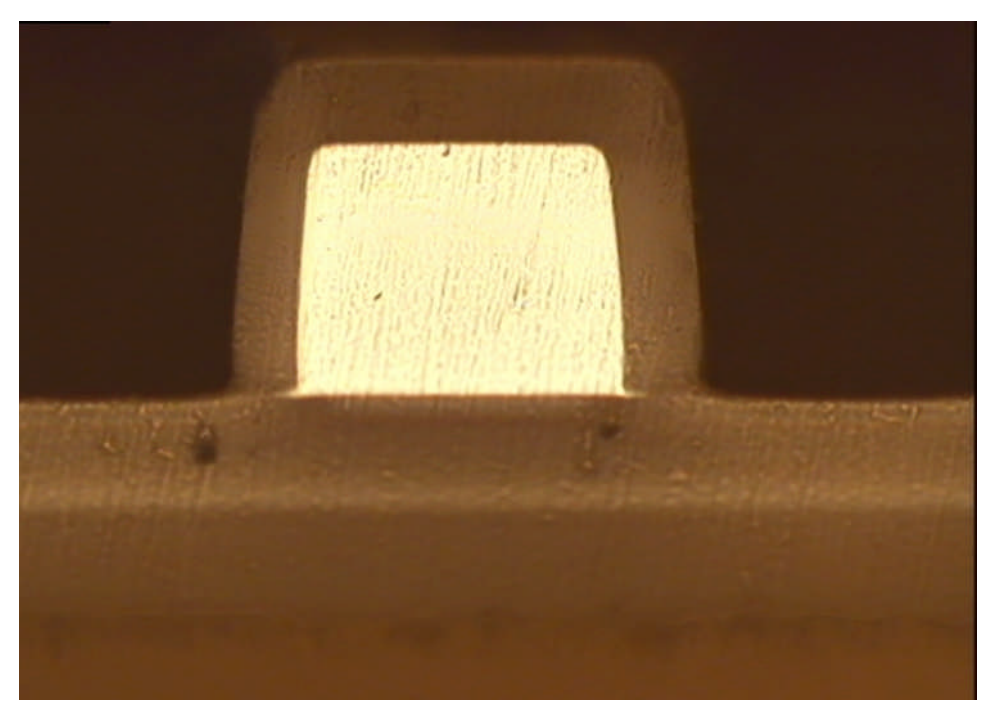

Figure 3: Cross-section of first order nested core waveguide illuminated with white light

\subsection{Fabrication defects}

The fabrication of nested core waveguides by photolithography is made significantly more complex by the need to very accurately align the secondary mask to the first waveguides. In spite of mounting of the stages on a highly accurate micro-stage, it was difficult to achieve a fully symmetric distribution. The waveguide test samples included nested core waveguides with an HCSK of $5 \mu \mathrm{m}$. However symmetric arrangement of the secondary cladding around the cores proved to be unachieveable given the alignment tolerances of the mask stage, thus giving rise to nested core waveguides where only one side of the core was covered with cladding, leaving the other side variably exposed along the axis of the waveguide. This in turn caused significant NA variations and consequently greater modal losses along the waveguide. Consequently measurements on $60 \mu \mathrm{m}$ nested core waveguides consistently yielded worse performance than the nested core waveguides with larger CSKs and the fully cladded waveguides. Some of the $70 \mu \mathrm{m}$ nested core waveguides also exhibit higher loss than expected, however less frequently due to the same effect i.e. occasional exposure of one of the core sides along the waveguide axis.

Additional defects were recently discovered on the nested core waveguides in the form of clumps of cladding residue lodged on the exposed sidewalls of the nested core waveguides. These proved to act as powerful scattering points giving rise to higher measured losses in all the nested core waveguides than expected. This will be mitigated in future research activities. 


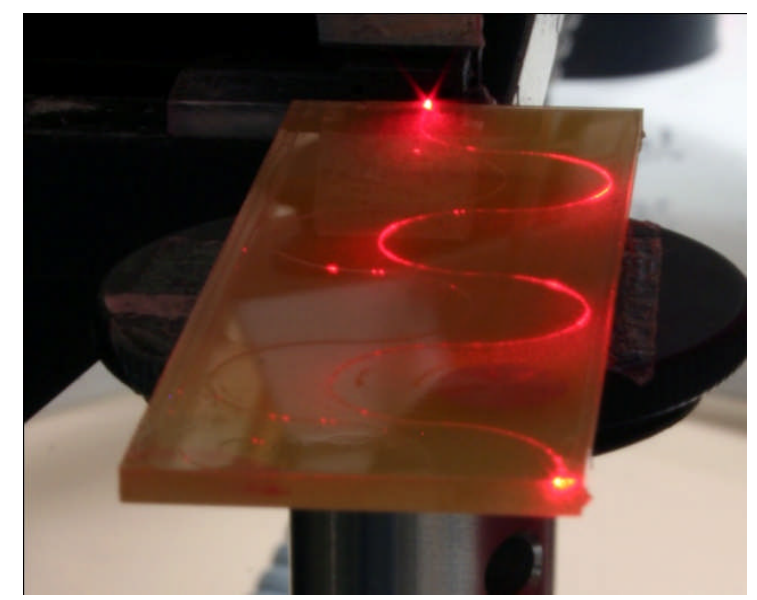

Figure 4: Nested core cascaded bend illuminated by $650 \mathrm{~nm}$ light. Scattering point defects are clearly visible along the waveguide axis and on the adjacent waveguide

\section{COMPARATIVE SIMULATION OF NESTED CORE WAVEGUIDE PERFORMANCE}

\subsection{Cascaded bend model}

Selected nested core waveguide structures were modeled and simulated using the BeamPROP optical simulation software from RSoft based on the finite difference beam propagation method (BPM).
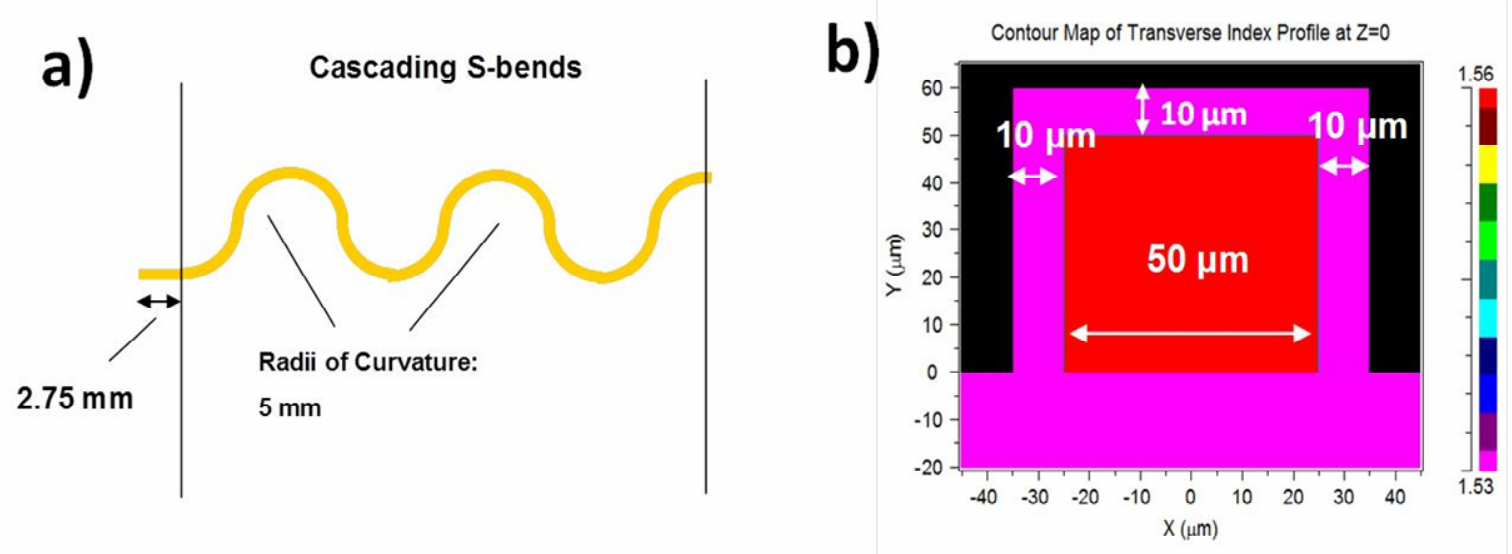

Figure 5: a) Model of multimode waveguide comprising 10 cascaded bends. b) Cross-section of nested core waveguide with Horizontal Cladding Skin Depth of $10 \mu \mathrm{m}$, Vertical Cladding Skin Depth of $10 \mu \mathrm{m}$, LIC of 0.03, HIC of 0.53

Figure 5a) shows one of the waveguide test patterns designed and fabricated to demonstrate stressed bend transition losses. The structure simulated consisted of 1 short straight section $(2.75 \mathrm{~mm})$ followed by 5 cascading circular Sbends (or 10 quarter circle arcs) of radius of curvature $5 \mathrm{~mm}$. Each $90^{\circ}$ bend (save the first bend) has an opposite sign of curvature to the previous bend, therefore creating a strong NA mismatch between each bend junction and consequently maximizing expected bend transition losses. The total length of the waveguide is $81.29 \mathrm{~mm}$.

Figure 5b) shows the cross-section of a 3D model of a nested core waveguide in the BPM program. The core refractive index is chosen as 1.56 and the cladding index 1.53 , which is consistent with the polymer materials used to fabricate the test structure. These give rise to an LIC value of 0.03 (i.e. $\mathrm{n}_{\text {core }}-\mathrm{n}_{\text {cladding }}$ ) and an HIC value of 0.53 (i.e. $\mathrm{n}_{\text {cladding }}-$ $\mathrm{n}_{\text {air }}$ ). The cladding skin depth is chosen as $10 \mu \mathrm{m}$, which relates to a structured cladding width of $70 \mu \mathrm{m}$, symmetrically arranged around the original $50 \mu \mathrm{m}$ core. 


\subsection{Comparative simulation results}

Comparative simulations of optical propagation along the cascaded bend test model outlined in Figure 5a were carried out for both a conventional multimode fully cladded waveguide with a core size of $50 \mu \mathrm{m}$ and for a nested core waveguide with the cross-sectional features shown in Figure 5b. The optical launch stimulus had a wavelength of 850 $\mathrm{nm}$ and a simple (fundamental) Gaussian mode profile of $50 \mu \mathrm{m}$ diameter, which was aligned to the centre of the waveguide core.

Propagation of optical power through the fully cladded waveguide (Figure 6a) showed distinct stepped power drops during transition between bend segments as expected, giving rise to an overall insertion loss of $1.9 \mathrm{~dB}$. On the nested core waveguide (Figure 6b), the transition bend losses are clearly reduced compared to that of the fully cladded waveguide and an insertion loss of $0.76 \mathrm{~dB}$ is projected, thereby predicting a comparative improvement in optical power transmission of $31.25 \%$ when using the selected nested core waveguide structure over a conventional fully cladded waveguide.

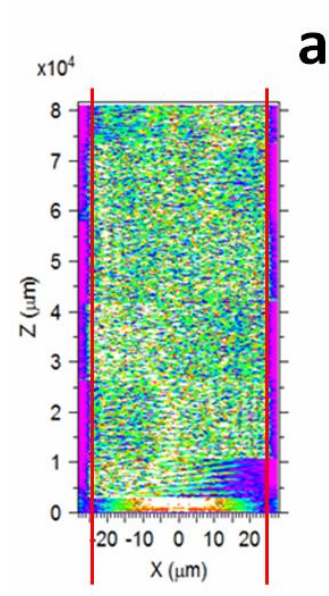

a)

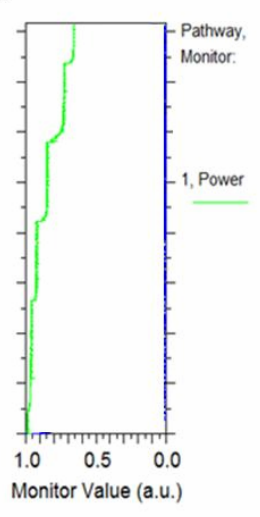

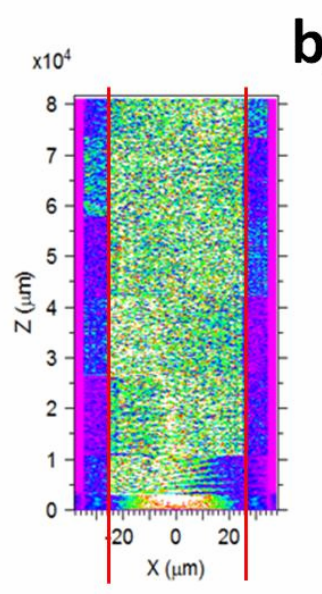

b)

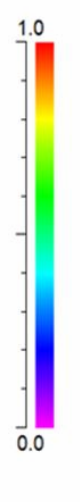

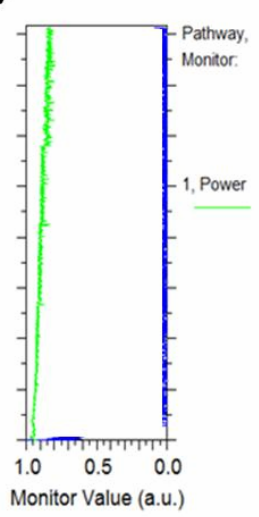

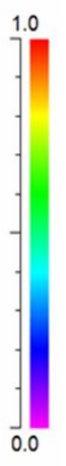

Figure 6: a) Simulated power loss during propagation through conventional waveguide (fully cladded) b) Propagation losses along nested core waveguide.

\section{EXPERIMENTAL CHARACTERISATION OF NESTED CORE WAVEGUIDES}

\subsection{Measurement set-up}

An MT - LC fibre-optic patch-cord comprised of $62.5 \mu \mathrm{m}$ graded index multimode fibre of 1 metre length was used to connect a commercial LC compliant $850 \mathrm{~nm}$ source to a flat MT ferrule, the output facet of which was aligned and butt-coupled to the waveguide end facets of the samples under test. The output power measured from the multimode fiber was $-1.67 \pm 0.03 \mathrm{dBm}$. A photo-detector (PD) with a $70 \mu \mathrm{m}$ diameter circular pinhole was placed at the output side of the waveguide to measure the insertion loss, which included the input facet coupling loss between launch fibre and the waveguide, propagation loss and the end facet coupling loss between waveguide and PD. Both the input source and PD were mounted on high precision xyz translation stages for accurate alignment. The launch fibre and PD aperture positions were optimized for maximum optical power transmission through the waveguide under test.

\subsection{Straight waveguide arrays}

Test groups of fully cladded and nested core straight multimode waveguide arrays of length $11.23 \mathrm{~cm}$ with a centre to centre pitch of $250 \mu \mathrm{m}$ were measured, whereby each group contained 6 parallel waveguides. The core sizes of both the fully cladded and nested core waveguides were $50 \mu \mathrm{m} \times 50 \mu \mathrm{m}$. Separate groups of nested core waveguide arrays with full (secondary) waveguide widths of $70 \mu \mathrm{m}(\mathrm{CSK}=10 \mu \mathrm{m}), 80 \mu \mathrm{m}(\mathrm{CSK}=15 \mu \mathrm{m})$ and $90 \mu \mathrm{m}(\mathrm{CSK}=20 \mu \mathrm{m})$ were characterised along with a group of fully clad waveguide arrays. The insertion loss as a function of full waveguide width is plotted in Figure 7 and shows little variation between the fully cladded waveguides and $80 \mu \mathrm{m}$ and $90 \mu \mathrm{m}$ nested core waveguides with the fully cladded waveguides exhibiting the lowest insertion loss. The $70 \mu \mathrm{m}$ 
nested core waveguides exhibit higher loss than expected. This may be due to asymmetric arrangement defects in the cladding as mentioned in section 2.3.

Measurement of input transverse misalignment tolerance was then carried out by varying the position of the launch fibre from its optimal position in $2 \mu \mathrm{m}$ steps over a full range of $200 \mu \mathrm{m}$ and recording variation in power received at the PD. We define power drop as the power recorded when the launch fibre was aligned to the waveguide core/cladding boundary. The higher the power drop at the core/cladding boundary, the higher the modal confinement in the core, which in turn relates to a smaller misalignment tolerance. The power drop in $\mathrm{dB}$ was plotted for each waveguide type. Both the fully cladded and nested core waveguides were characterised and plotted in Figure 7 in terms of the power drop at the core cladding boundary. The measurements yielded a lower input misalignment tolerance (higher confinement in the core) for the nested core waveguides than for the fully cladded waveguides, which would make it more difficult to align to nested core waveguides.

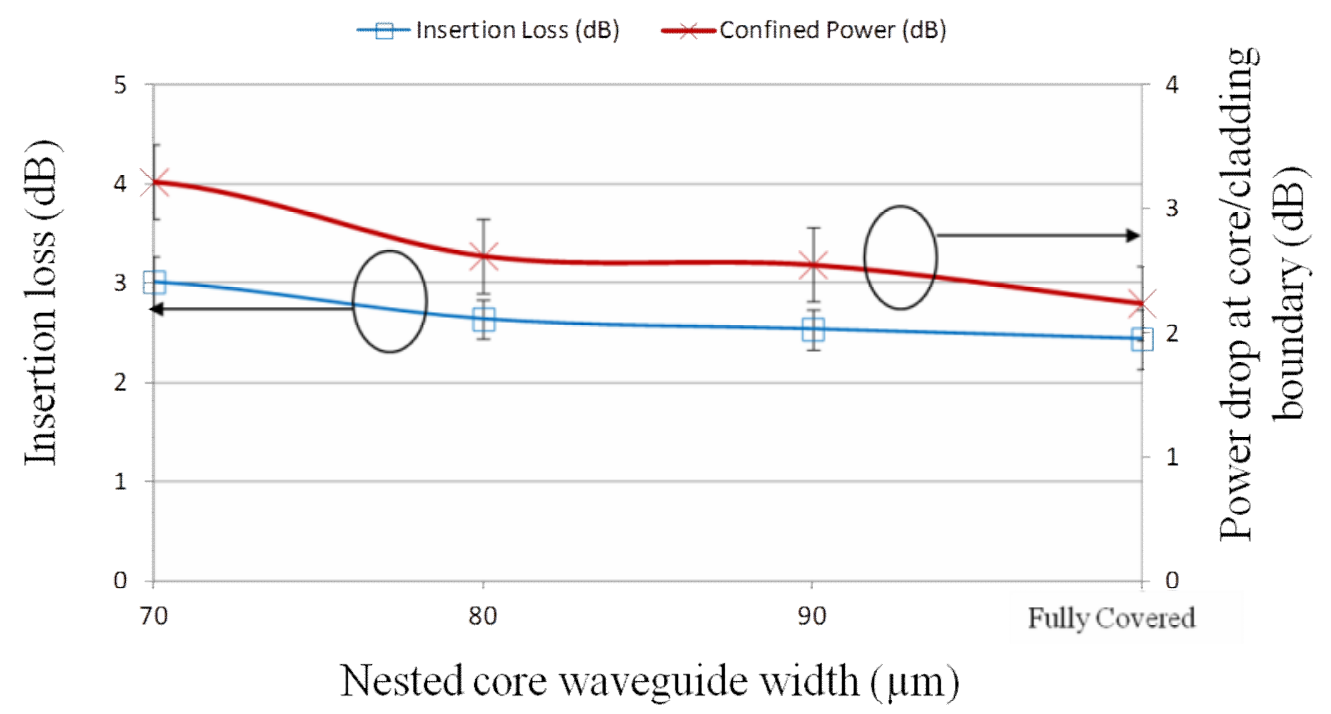

Figure 7: Measurement results for insertion loss and power drop at the core cladding boundary for fully cladded and $70 \mu \mathrm{m}$, $80 \mu \mathrm{m}, 90 \mu \mathrm{m}$ bested waveguides.

\subsection{Cascaded waveguide measurements}

Insertion loss measurements were carried out on $70 \mu \mathrm{m}, 80 \mu \mathrm{m}$ and $90 \mu \mathrm{m}$ nested core and fully cladded cascaded waveguide samples, the layout of which is described in section 3.1 and shown in Figure 5a. The average insertion loss based on 5 repeated measurements is plotted for each waveguide type in Figure 8. Again there is little variation in measured insertion loss between the waveguide samples with the $80 \mu \mathrm{m}$ nested core waveguide showing the least insertion loss. 


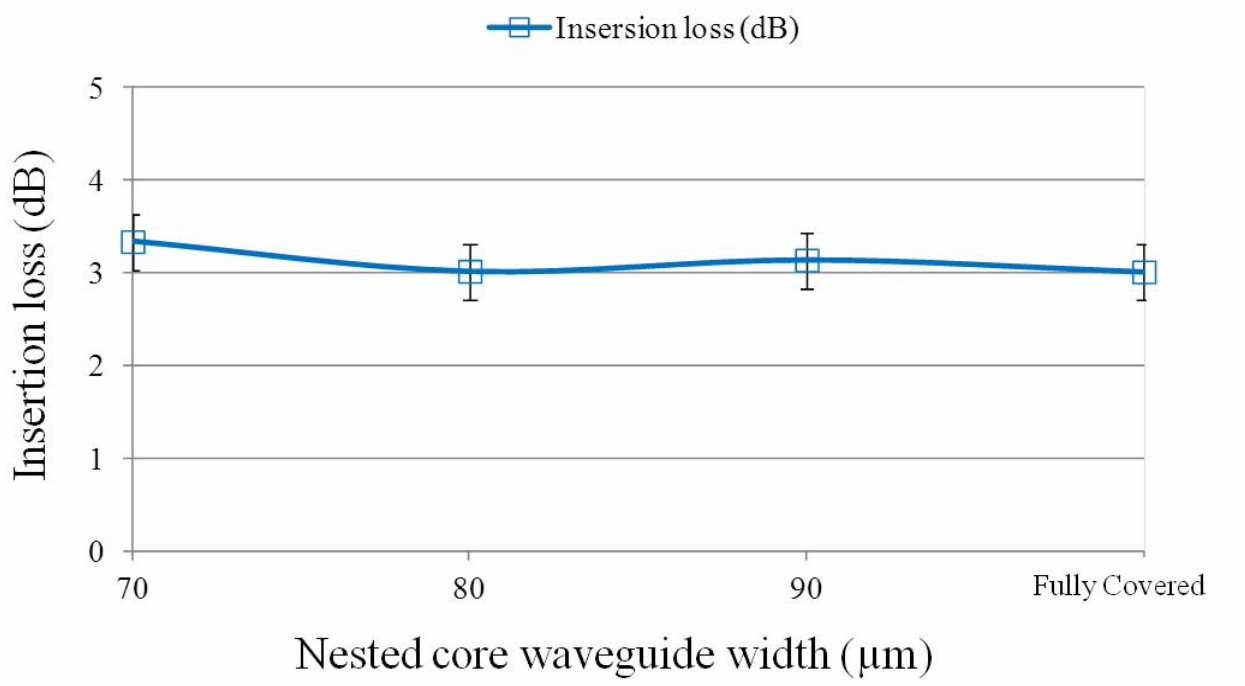

Figure 8: Insertion loss (including input and output coupling losses) measured for 4 waveguides with 10 cascaded 5 mm radius $90^{\circ}$ bends with different cladding widths varying from $70 \mu \mathrm{m}, 80 \mu \mathrm{m}, 90 \mu \mathrm{m}$ to fully cladded

\section{CONCLUSIONS AND FUTURE WORK}

Though simulations of $1^{\text {st }}$ order nested core waveguides predict a $1.14 \mathrm{~dB}$ reduction in transition bend losses, the results of comparative experimental characterisation of nested core waveguides with fully cladded waveguides are as yet inconclusive. It should be noted however that this is the first time such multimode structures have been fabricated and due to the complexity of fabrication, various defects have emerged, which would be expected to reduce the performance of the nested core waveguides under test. These include asymmetric and variable arrangement of the structured cladding along the axis of the waveguide, side wall roughness of the outer HIC boundary and residue defects lodged on the exposed side walls as shown in Figure 4. This research activity is currently entering a new phase, for which new nested core waveguide samples have been produced using a different fabrication technique based on laser direct imaging. This new technique is expected to give rise to side walls of higher quality, better symmetry and less defects. In addition some nested core samples will be coated with a further lower refractive index cladding (to replace air) to allow evaluation of nested core waveguides with lower HIC values.

\section{REFERENCES}

[1] D. R. Selviah et al., "Integrated Optical and Electronic Interconnect PCB Manufacturing Research,” Circuit World, vol. 36, no. 2, pp. 5-19, 2010 .

[2] D. R. Selviah, "Polymer multimode waveguide optical and electronic PCB manufacturing," Proceedings of SPIE, pp. 721905-721905-10, 2009 .

[3] R. Pitwon et al., Design and implementation of an electro-optical backplane with pluggable in-plane connectors. SPIE-INT SOC OPTICAL ENGINEERING, 2010, p. 76070J-76070J-12.

[4] I. Papakonstantinou, K. Wang, D. R. Selviah, and F. A. Fernandez, "Transition, radiation and propagation loss in polymer multimode waveguide bends," OPTICS EXPRESS, vol. 15, no. 2, pp. 669-679, 2007.

[5] K. Wang, D. R. Selviah, I. Papakonstantinou, H. Baghsiahi, and F. A. Fernandez, "Photolithographically manufactured acrylate polymer multimode optical waveguide loss design rules," in 2008 2nd Electronics Systemintegration Technology Conference, 2008, pp. $1251-1256$.

[6] D. a. Roberts, M. Rahm, J. B. Pendry, and D. R. Smith, “Transformation-optical design of sharp waveguide bends and corners,” Applied Physics Letters, vol. 93, no. 25, p. 251111, 2008.

[7] H. Baghsiahi, D. R. Selviah, M. Yau, and F. A. Fernandez, "Photolithographically manufactured acrylate multimode optical waveguide translation and rotation misalignment tolerances," in 2008 2nd Electronics Systemintegration Technology Conference, 2008, pp. 617-622.

[8] I. Papakonstantinou, D. R. Selviah, R. Pitwon, and D. Milward, "Low-Cost, Precision, Self-Alignment Technique for Coupling Laser and Photodiode Arrays to Polymer Waveguide Arrays on Multilayer PCBs," IEEE Transactions on Advanced Packaging, vol. 31, no. 3, pp. 502-511, Aug. 2008. 
[9] I. Papakonstantinou, R. James, and D. R. Selviah, "Radiation- and Bound-Mode Propagation in Rectangular, Multimode Dielectric , Channel Waveguides With Sidewall Roughness," Lightwave, vol. 27, no. 18, pp. 4151-4163, 2009.

[10] C. Berger, "Challenges for introducing board-level optical interconnects into product development roadmaps," International Symposium on Photonic Packaging, 2006.

[11] S. Uhlig et al., "Polymer Optical Interconnects — A Scalable Large-Area Panel Processing Approach," Online, vol. 29, no. 1, pp. 158-170, 2010 .

[12] N. Bamiedakis et al., "Cost-Effective Multimode Polymer Waveguides for High-Speed On-Board Optical Interconnects," Quantum, vol. 45, no. 4, pp. 415-424, 2009.

[13] R. Barbieri et al., "Design and construction of the high-speed optoelectronic memory system demonstrator.," Applied Optics, vol. 47, no. 19, pp. 3500-12, Jul. 2008. 